\section{Drift velocities and characteristic energies of electrons in deuterium at low and moderate $\mathrm{E} / \mathrm{N}$}

To cite this article: W Roznerski et al 1994 J. Phys. D: Appl. Phys. 272060

View the article online for updates and enhancements.
Related content

- $\frac{\mathrm{e}^{-} \text {-Ar scattering length from drift velocities }}{\text { measured in argon-hydrogen mixtures }}$
Z L Petrovic, T F O'Malley and R W
Crompton
- Review Article
Z Lj Petrovi, S Dujko, D Mari et al.
- Electron drift velocity in hydrogen,
$\frac{\text { nitrogen, oxygen, carbon monoxide. }}{\text { carbon dioxide and air at moderate E/N }}$
W Roznerski and K Leja

\section{Recent citations}

- Scanning drift tube measurements of
$\frac{\text { electron transport parameters in different }}{\text { gases: argon, synthetic air, methane and }}$
$\frac{\text { deuterium }}{\text { I Korolov et al }}$
- Measurement and interpretation of swarm
parameters and their application in plasma
$\frac{\text { modelling }}{Z \text { Lj Petrovi et al }}$
- $\frac{D_{f} / \mu \text { and } D_{,} / \mu \text { for electrons in } N O}{\text { Jadwiga Mechlinska-Drewko et al }}$




\title{
Drift velocities and characteristic energies of electrons in deuterium at low and moderate $E / N$
}

\author{
W Roznerski†, J Mechlińska-Drewko†, $\mathrm{K}$ Leja† and \\ Z Lj Petrovićł§ \\ † Institute of Physics, Technical University of Gdańsk, 80-952 Gdańsk, Poland \\ $\ddagger$ Institute of Physics, University of Belgrade, PO Box 68, 11080 Zemun, \\ Yugoslavia
}

Received 20 April 1994, in final form 4 July 1994

\begin{abstract}
The measurements of the drift velocities and characteristic energies of electrons in deuterium are presented. Drift velocities are obtained between 3 and 125 Td by a standard Bradbury-Nielsen technique with a double set of shutters. The characteristic energy for the transverse diffusion was measured by a standard Townsend-Huxley technique to be between 3 and $1000 \mathrm{Td}$.

The drift velocities and characteristic energies are in good agreement with the best available data in the range of overlap and extend the availability of the experimental data to higher $E / N$. The predictions based on the best available cross section sets do not match the experimental results at higher $E / N$ above the limits of the previously available data.
\end{abstract}

\section{Introduction}

Electron scattering cross sections are of great importance in a wide range of applications. Modelling of plasma processing devices is most frequently cited as the major application of cross section data (Morgan 1992).

Specifically, the cross sections for electron-hydrogen and electron-deuterium scattering are required for the understanding of a number of plasma devices and processes such as thyratrons (Schaefer et al 1990, Kushner 1988) and pseudo sparks (Gundersen and Schaefer 1990); tokamak start-up (Fujiwara et al 1983, Buffa et al 1971); plasma etching and cleaning (Kuroda and Iwakuro 1993, Tomzik et al 1987); plasma deposition, negative ion generators for fusion and other applications (Hiskes 1980) and also for the interpretation of a number of atmospheric and astrophysical phenomena.

Fundamental knowledge of total cross sections is often overlooked. Calculation of the cross sections involves some of the greater intellectual challenges and there are still many unresolved issues. The crucial test case for the theory is that of electron-hydrogen molecule scattering which involves the smallest number of electrons, and the largest gaps between the rotationally excited and vibrationally excited levels.

$\S$ Also at MTT INFIZ and Faculty of Electrical Engineering, University of Belgrade, Yugoslavia.
Deuterium is also an important case for both theory and experiment, since similar theoretical techniques may be applied to both hydrogen and deuterium. The problems that still remain is disagreement between some experimental and theoretical data for the cross sections for the vibrational excitation of hydrogen (Morrison et al 1987, Petrovic and Crompton 1987, England et al 1988, Crompton and Morrison 1993, Schmidt 1993, Morgan 1993, Buckman et al 1990) should also be present in the case of deuterium. Thus, deuterium may be an important test for extending some electron scattering theories from hydrogen to heavier molecules (Bardsley and Wadehra 1979, Chang 1974, Henry and Lane 1971). Experimental studies of isotope effects, especially in the case of hydrogen, thus provide important guidance for the theorists besides their importance in various applications (Chang and Wong 1977, Carnahan and Zipf 1977, de Heer 1981).

Transport coefficients for electron swarms are especially useful to establish the electron scattering cross sections at low energies (Crompton 1969). While interpretation of such data may not be as straightforward as in the crossed beam experiments, the results obtained under conditions when understanding of the swarm experiment is complete and the cross section analysis can give unique results are often of the higher accuracy.

In addition, some theoretical techniques for modelling gas discharges rely on direct application of the electron transport data. Knowledge of characteristic energies and drift velocities is important in breakdown 
strength studies and the isotope dependence for the case of hydrogen and deuterium has been studied by Christophorou et al (1983). Recent advances in the understanding of DC (Phelps et al 1993, Petrovic and Phelps 1993, Petrovic et al 1992, Ganguly and Garscadden 1991, Barbeau and Jolly 1990, Vrhovac et at 1991) and RF (Kokubo et al 1989) discharges in hydrogen rely on the accurate knowledge of the electron transport $\mathrm{cO}$ efficients for moderate and high $E / N$ range.

Drift velocity $v_{\mathrm{dr}}$ measurements for detterium were performed by Pack et al (1962), McIntosh (1966), Crompton et al (1968), Robertson (1971) and Crompton and Robertson (1971). Drift velocity in $\boldsymbol{E} \times \boldsymbol{B}$ experiments together with the magnetic deflection coefficient was measured for deuterium by Creaser (1967).

Characteristic energy $\left(e D_{\mathrm{T}} / \mu\right)$ measurements were performed by Hall (1955), Warren and Parker (1962), McIntosh (1966) and Crompton et al (1968). It is of interest to note that Rhymes (1976) found the thermal diffusion coefficient to be the same for hydrogen and deuterium indicating that the low-energy momentum transfer cross section is the same.

Engelhardt and Phelps (1963) used early transport data to obtain a set of electron scattering cross sections. Gibson (1970) used a set of high-accuracy transport coefficients measured at Australian National University to obtain an improved set of low-energy cross sections. The energy range where the cross sections could be determined overlapped only with the threshold region of the vibrational excitation cross section. Buckman and Phelps (1985) measured excitation coefficients for the first vibrationally excited state of deuterium by applying a tracer technique. $\mathrm{CO}$ and $\mathrm{CO}_{2}$ were used as tracer gases. Vibrational excitation coefficients together with earlier transport data were the basis of the cross section set obtained by Buckman and Phelps (1985).

These authors however could not obtain a good fit to both the vibrational excitation rate coefficients and the transport data. Two sets of cross sections have been proposed. The characteristic energies obtained with the set that fits the excitation rates best were in disagreement with the only available data of Hall (1955) in the relevant $E / N$ range. As the data of Hall were not obtained with the modern, high-accuracy experimental techniques, Petrović and Crompton (1989) extended the characteristic energy measurements to $100 \mathrm{Td}(3-100 \mathrm{Td})$. They also extended the drift velocity measurements to $30 \mathrm{Td}(3-30 \mathrm{Td})$. Their measurements confirmed the results of Hall (1955) and thus the disagreement with excitation rates has not been resolved. The experimental apparatus of Petrović and Crompton (1989) is of the highest presently available accuracy.

The aim of the present paper is to verify the data of Petrovic and Crompton (1989) in the overlapping energy range and to extend the data to higher $E / N$ which is of interest in many applications and for testing the cross sections of Buckman and Phelps (1985). Preliminary presentation of these data was made by Roznerski et al (1989).

\section{Experiment}

\subsection{Apparatus}

The double grid Bradbury-Nielsen system was used for the drift velocity measurements (Huxley and Crompton 1974, Blevin and Hasan 1967). Drift distance between the shutters was $10.97 \mathrm{~cm}$. RF voltage was applied to the shutters with frequencies between $20 \mathrm{kHz}$ and $20 \mathrm{MHz}$ and with the amplitude of $0-21 \mathrm{~V}$. The current transmitted through both sets of shutters is monitored as a function of frequency once the amplitude has been properly adjusted. Thus the time of fight spectrum is converted to the frequency dependence of the transmitted current. The source of electrons was UV illuminated gold leaf photocathode. The apparatus for measuring drift velocities has been described in greater detail by Roznerski and co-workers (Roznerski and Leja 1984).

Characteristic energies were measured in a TownsendHuxley type apparatus (Huxley and Crompton 1974) which has been described in more detail (Roznerski and Mechlinska-Drewko 1977, 1979, Roznerski 1979, Roznerski and Leja 1980, Roznerski et al 1990, Braglia et al 1984). In brief, a point source releases thermal electrons that drift and diffuse in $h=9.88 \mathrm{~cm}$ long drift chamber. Electron current is measured at two circular segments of the anode, the central circle with radius $b=1.268 \mathrm{~cm}$ and the annular part with the outer radius of $c=4.964 \mathrm{~cm}$. The homogeneous field due to voltage applied between the anode and the cathode is maintained by a set of guard rings with an inner diameter of $10 \mathrm{~cm}$.

Both drift tubes were baked and evacuated to a low background pressure. The procedure gave negligible rate of rise of the pressure. Research grade purity (Matheson) deuterium was used in experiments without further purification. Deuterium, being a molecular gas, is not affected strongly by small amounts of impurities as in the case of rare gases (Huxley and Crompton 1974). Pressure was measured by a McLeod gauge connected with liquid nitrogen or the dry ice traps and the range between 7 and 0.07 Torr was used in the present measurements. All measurements were performed at room temperature of $294.5 \pm 1.5 \mathrm{~K}$ and the temperature variation during the measurements was less than $0.5 \mathrm{~K}$.

\subsection{Procedure}

The measurements of the drift velocity have a straightforward interpretation. The frequency is converted to the time of flight directly. The results at each pressure are an average of several repeated measurements. The boundary effects due to shutters and diffusion in the shutter region are small and no systematic trends larger than the statistical scatter were observed.

The transverse characteristic energy $\left(e D_{\mathrm{T}} / \mu\right)$ measurements have been performed following the procedure outlined before (Braglia et al 1984) ( $D_{\Upsilon}$ is the transverse diffusion coefficients while $\mu$ is the electron mobility). 
The currents to the two segments of the anode were always less than $1 \times 10^{-12} \mathrm{~A}$ and have been measured by the two calibrated electrometers.

The electron number density in the TownsendHuxley experiment can be described by an infinite dipole extension formula (Warren and Parker 1962, Lowke 1973, Huxley and Crompton 1974):

$$
n(z, \rho)=\sum_{k=-\infty}^{k=+\infty} r_{k}^{-3}(z-2 k h)\left(\beta r_{k}+1\right) \exp \left(\lambda_{\mathrm{L}} z-\beta r_{k}\right)
$$

where

$$
\begin{gathered}
r_{k}=\left[(z-2 h k)^{2}+\left(D_{\mathrm{L}} / D_{\mathrm{T}}\right) \rho^{2}\right]^{1 / 2} \\
\beta=\lambda_{\mathrm{L}}\left(1-2 \alpha / \lambda_{\mathrm{L}}\right)^{1 / 2} \quad \lambda_{\mathrm{L}}=\frac{v_{\mathrm{dr}}}{2 D_{\mathrm{L}}}
\end{gathered}
$$

and $\alpha$ is the Townsend ionization coefficient, $z$ is the distance from the cathode, $\rho$ is the radius in the cylindrical coordinate system, and $D_{\mathrm{L}}$ and $D_{\mathrm{T}}$ are the longitudinal and transverse components of the diffusion tensor respectively.

From the expression for the electron number density one can obtain the expression for the distribution of the current as a function of radial dimension and distance from the cathode:

$$
\begin{aligned}
& i(z, \rho)=-2 \pi D_{\mathrm{T}} \sum_{k=-\infty}^{k=+\infty}\left[r_{k}^{-3} \exp \left(\lambda_{\mathrm{L}} h-\beta r_{k}\right)\right. \\
& \left.\quad \times\left\{\left(\beta r_{k}+1\right)(2 k-1)^{2} h^{2}-\left[1-\lambda_{\mathrm{L}} h(2 k-1)\right] r_{k}^{2}\right\}\right]_{\rho_{1}}^{\rho_{1}}
\end{aligned}
$$

where $\rho_{1}$ and $\rho_{2}$ are the inner and outer radii of any annular area of the electrode respectively.

Measurements are performed as a function of $E / N$ at several $N$. The unit adopted here for $E / N$ is one Townsend (Td) which is $10^{-21} \mathrm{~V} \mathrm{~m}$ (Huxley et al 1966). In addition, measurements are made for different selections of the radii of the sections of the segmented anode. The fraction of the current to the central circular segment of the anode can be under special circumstances (Huxley and Crompton 1974) described by the Lowke formula corrected for ionization effects. For the conditions of our experiment both $D_{\mathrm{L}} / D_{\mathrm{T}} \neq 1 / 2$ and $\alpha_{\mathrm{T}} \neq 0$ thus the formula cannot be reduced to the Huxley formula (Huxley and Crompton 1974).

Petrovic and Crompton (1989) found that applying the Huxley formula gave results for $D_{\mathrm{T}} / \mu$ that were not dependent on the pressure while the application of the Lowke formula gave somewhat different (less than $2 \%$ ), but systematically pressure dependent, results. These authors used calculated values of $D_{\mathrm{L}} / D_{\mathrm{T}}$ in their analysis. In this paper we use $D_{\mathrm{L}}$ as a fitting parameter to remove the pressure dependence of the results. Thus $D_{\mathrm{L}} / \mu$ can be obtained to an accuracy of $\pm 10 \%$ with the resulting overall uncertainty of $\pm 5 \%$ for $D_{\Upsilon} / \mu$. In our calculations we also use the available data for the $D_{\mathrm{L}} / D_{\mathrm{T}}$ ratio (Lowke and Parker 1969). Where no data are available we use the $D_{\mathrm{L}} / D_{\mathrm{T}}$ ratio calculated from the multi-term calculations (Petrović 1985), analytical formulae based on momentum transfer theory (Robson 1972) and the data for the ratio for hydrogen (Roznerski et al 1990). For $E / N$ greater than $30 \mathrm{Td}$ the ratio for deuterium is close to that in hydrogen to a few per cent which converts to a very small uncertainty in the obtained $D_{\mathrm{T}} / \mu$. When $D_{\mathrm{L}}$ was used as the fitting parameter for the measurements where sufficiently accurate value of the $D_{\mathrm{L}} / D_{\mathrm{T}}$ ratio could be obtained results agreed well with the input data based on the hydrogen results.

At the highest $E / N$ and correspondingly the lowest pressures, non-equilibrium effects could affect the electron swarm throughout a large part of the drift distance. Therefore we make measurements at several pressures to make sure that the results are not affected. The range of pressures covered above $400 \mathrm{Td}$ is below 1:2 but is larger than 1:1.6 except for $1000 \mathrm{Td}$. The small pressure dependence is attributed to the effect of longitudinal diffusion and the resulting correction agrees well with the correction for hydrogen. From our experience with low-pressure discharges (Petrovic et al 1992, Stokic et al 1992) in hydrogen and deuterium and from the preliminary results of our Monte Carlo simulations we expected that the upper end of our $E / N$ range coincides with the onset of significant nonequilibrium effects for the geometry and pressures used in our measurements and that still at $1000 \mathrm{Td}$ the effect is small and, for lower $E / N$ values, negligible. Nevertheless, extension of the measurements of transport coefficients in hydrogen and deuterium to $E / N>$ $1000 \mathrm{Td}$ should be done with caution.

Ionization coefficients of Rose (1956) were used in the analysis. The maximum value of the ionization correction $\alpha_{\mathrm{T}} / a_{\mathrm{L}}$ is $18.5 \%$ at $1000 \mathrm{Td}$. The correction is gradually reduced with $E / N$ and is less than $1 \%$ below $100 \mathrm{Td}$. The corresponding uncertainty of $D_{\mathrm{T}} / \mu$ due to the claimed uncertainty of $\pm 2 \%$ for $\alpha_{\mathrm{T}}$ (Rose 1956) is $0.3 \%$. The ionization coefficient of Rose for deuterium is in reasonably good agreement with the yet unpublished high-accuracy data of Haydon and co-workers (1984) over most of the relevant $E / N$ range.

No significant systematic pressure dependences were observed for both $v_{\mathrm{dr}}$ and $e D_{\mathrm{T}} / \mu$. The measurements for $E / N$ from 3-30 Td were performed between 1 and 7 Torr, from $30 \mathrm{Td}-125 \mathrm{Td}$ between 1 Torr and 0.3 Torr and for 125 and $1000 \mathrm{Td}$ between 0.3 and 0.06 Torr.

\section{Results and discussion}

\subsection{Drift velocities}

Results of our measurements of drift velocities in the $E / N$ range between 3 and $125 \mathrm{Td}$ are shown in figure 1 together with some of the other available data and in table 1 . The present results are somewhat larger than the data of Petrovic and Crompton (1989) but the difference is less than the error bounds of the more accurate apparatus of Petrovic and Crompton. This good agreement gives us confidence in the higher $E / N$ data. 


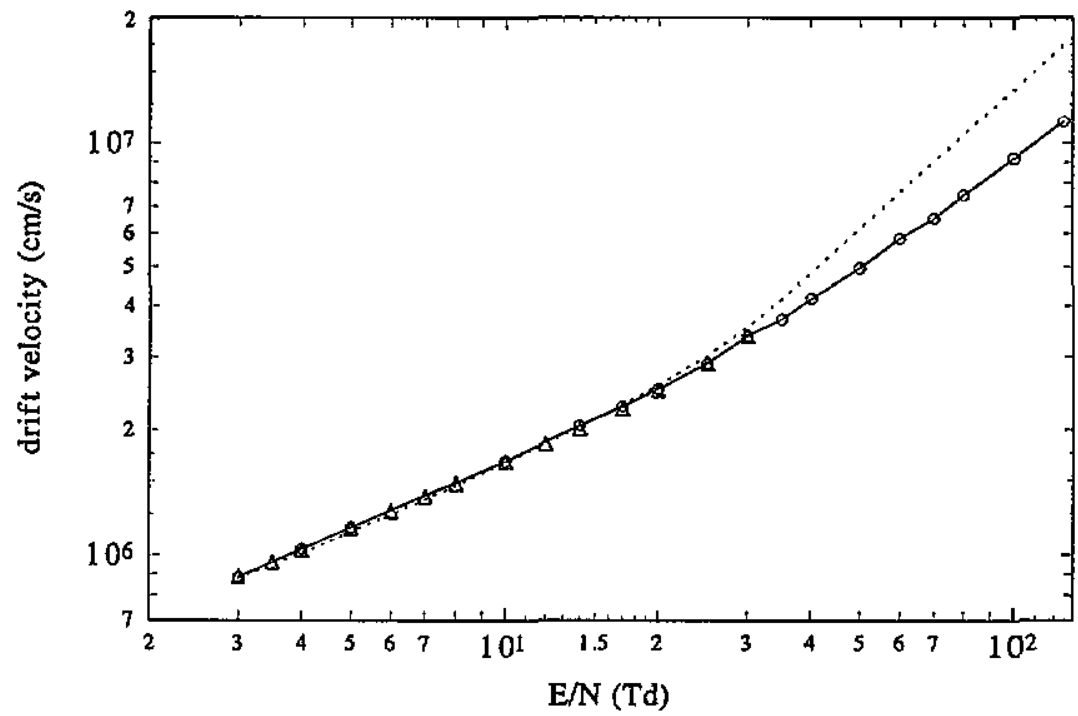

Figure 1. Drift velocities in deuterium. Data of Petrović and Crompton (1989) are given by triangles, present results by open circles and a full circle and the predictions based on the 'reference' set of Buckman and Phelps (1985) by a dotted curve.

Table 1. Transport coefficients for electrons in deuterium.

\begin{tabular}{|c|c|c|}
\hline $\begin{array}{l}E / N \\
(\mathrm{Td})\end{array}$ & $\begin{array}{l}V_{\mathrm{dr}} \\
\left(\times 10^{5} \mathrm{~cm} \mathrm{~s}^{-1}\right)\end{array}$ & $\begin{array}{l}e D_{\top} / \mu \\
(\mathrm{eV})\end{array}$ \\
\hline $\begin{array}{r}3 \\
4 \\
15 \\
10 \\
14 \\
17 \\
20 \\
25 \\
30 \\
35 \\
40 \\
50 \\
60 \\
70 \\
80 \\
100 \\
125 \\
150 \\
175 \\
200 \\
250 \\
300 \\
350 \\
400 \\
500 \\
600 \\
750 \\
1000\end{array}$ & $\begin{array}{r}8.84 \\
10.35 \\
11.60 \\
16.75 \\
20.48 \\
22.82 \\
25.11 \\
29.01 \\
33.83 \\
37.17 \\
41.73 \\
49.59 \\
58.48 \\
65.39 \\
74.73 \\
91.96 \\
112.80\end{array}$ & $\begin{array}{r}0.274 \\
0.334 \\
0.388 \\
0.615 \\
0.773 \\
0.887 \\
1.026 \\
1.241 \\
1.474 \\
1.697 \\
1.876 \\
2.18 \\
2.33 \\
2.49 \\
2.63 \\
2.97 \\
3.16 \\
3.42 \\
3.72 \\
4.06 \\
4.64 \\
5.20 \\
5.80 \\
6.37 \\
6.86 \\
7.61 \\
9.31 \\
11.69\end{array}$ \\
\hline
\end{tabular}

At lower $E / N$ values our results agree very well with the data of McIntosh (1966) and the high $E / N$ and $77 \mathrm{~K}$ data agree with Crompton et al (1968).

As a more detailed comparison with the other sources of data has been presented by Petrovic and Crompton we shall only compare our data to theirs in this paper.

\subsection{Characteristic energies}

Our results for $e D_{\Upsilon} / \mu$ are shown in figure 2 and in table 1. Except at two $E / N$ values, the present $e D_{\mathrm{T}} / \mu$ results agree very well with those of Petrovic and Crompton (1989) to better than $\pm 1 \%$ and even at the two points in question the disagreement is just over $\pm 2 \%$ which is within the error bounds of the present data. The disagreement at $100 \mathrm{Td}$ increases to $3 \%$ when ionization correction is included in the analysis of Petrović and Crompton, while at all other $E / N$ in the overlapping region nothing changes with the inclusion of the correction. The agreement with other available data in the range of overlap (Hall 1955, McIntosh 1966, Crompton et al 1968) is also very good while the results of Warren and Parker differ from our data systematically by about $5 \%$ which may be within the combined error bounds but is not acceptable since it is systematic.

\subsection{Discussion}

The drift velocity data, whilst agreeing with other sources, disagree with the values on the basis of the cross sections of Buckman and Phelps (1985) for $E / N>$ $30 \mathrm{Td}$. The 'reference' and 'adjusted' sets of Buckman and Phelps give drift velocities that agree closely. The calculations have been performed by a two-term solution to the Boltzmann equation (Gibson 1970) and do not take into account the non-conservative transport (Brunet and Vincent 1979, Yoshida et al 1983).

The differences between the predicted and measured drift velocities can be easily overcome by adjusting the momentum transfer cross section correspondingly. However, one should make this adjustment in an attempt to make an overall agreement with other sources of data. Complete analysis should include the vibrational and electronic excitation coefficients, 


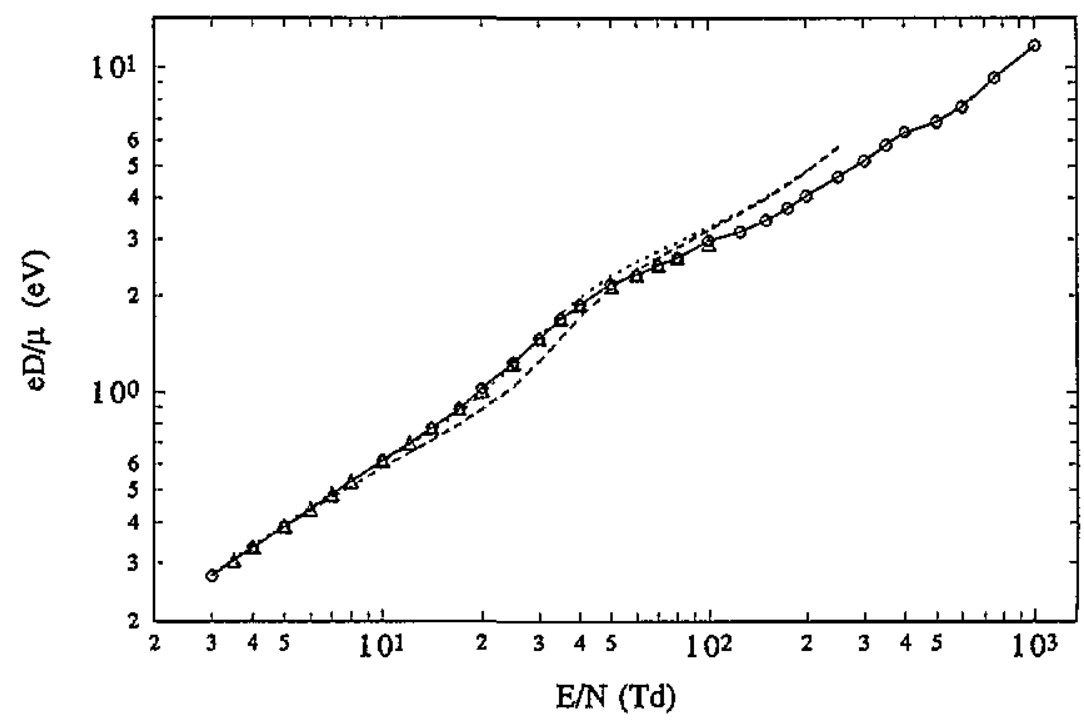

Figure 2. Characteristic energies in deuterium. Data for $e D_{\uparrow} / \mu$ of Petrović and Crompton (1989) are given by triangles, present results by open circles and a full curve and the predictions based on the 'reference' and 'adjusted' sets of Buckman and Phelps (1985) by dotted and broken curves respectively.

ionization coefficients and characteristic energies for both transverse and longitudinal diffusion together with an attempt to represent properly the non-conservative processes. Such an effort has failed for hydrogen so far (Buckman and Phelps 1985) to produce a unique set of cross sections and a new cross section analysis is outside the scope of this paper.

The present results confirm the data of Petrovic and Crompton (1989) (and the earlier data of Hall (1955)) in the overlapping $E / N$ range. Thus our data disagree with predictions of both the 'reference' and the 'adjusted' sets of Buckman and Phelps (1985). There is expected agreement with the 'reference' set below $30 \mathrm{Td}$, while the adjusted set agrees with our data only below $10 \mathrm{Td}$. On the other hand the 'reference' set cannot fit the excitation coefficients measured by Buckman and Phelps (1985). As the mean energies begin to overlap with the higher energy end of the swarm analysis, it becomes important to include the ionization and electronic excitation rate coefficients. This experiment, together with some recent measurements of electronic excitation coefficients (Stokic et al 1992), can provide the data needed to make the necessary adjustments, but the problem is not trivial and will require significant effort.

\section{Conclusion}

In this paper we have presented measurements of the drift velocities and characteristic energies in deuterium. Drift velocities are obtained between 3 and $125 \mathrm{Td}$ by a standard Bradbury-Nielsen technique with a double set of shutters and the data are obtained with an accuracy of between $\pm 1.5 \%$ in the lower $E / N$ range ( $<50 \mathrm{Td}$ ) and $\pm 3 \%$ at the highest $E / N$. The characteristic energy for the transverse diffusion was measured by a standard Townsend-Huxley technique between 3 and $1000 \mathrm{Td}$. The uncertainty for the characteristic energy ranges between 2 and 5\% between the lowest and the highest $E / N$.

The drift velocities and characteristic energies are in good agreement with the best available data in the range of overlap and extend the availability of the experimental data to higher $E / N$. In that range, however, the measured values are in disagreement with the predictions of the best available cross section sets thus warranting the reanalysis of the cross sections. Even with a more limited set of data, a unique set of cross sections could not be obtained (Buckman and Phelps 1985) so we hope that the present data will make it possible, together with some other newly obtained transport data, to make a unique cross section set.

\section{Acknowledgments}

This work was supported in part by Komitet Badań Naukowych project PB 1777/2/91. Z Petrovic is grateful to the Ministry of Science and Technology of Serbia and USA-Yugoslavia project JF 926 for partial support.

\section{References}

Barbeau C and Jolly J 1990 J. Phys. D: Appl. Phys. 231168

Bardsley J N and Wadehra J M 1979 Phys. Rev. A 201398

Blevin H A and Hasan M Z 1967 Aust. J. Phys. 20735

Braglia G L, Romano L and Roznerski W 1984 Beitr. Plasmaphys. 24113

Brunet $\mathrm{H}$ and Vincent P 1979 J. Appl. Phys. 504700

Buckman S J, Brunger M J, Newman D S, Snitchler G, Alston $S$, Norcross D W, Morrison M A, Saha B C, Danby G and Trail W K 1990 Phys. Rev. Lett. 653253 
Buckman S J and Phelps A V 1985 J. Chem. Phys. 824999 Buffa A, Malesani G and Nalesso G F 1971 Phys. Rev. A 3955 Carnahan B L and Zipf E C 1977 Phys. Rev. A 16991

Chang E S 1974 Phys. Rev. A 91644

Chang E S and Wong S F 1977 Phys. Rev. Lett. 381327

Christophorou L G, Mathis R A and James D R 1983 J. Appl. Phys. 543098

Creaser R P 1967 Aust. J. Phys. 20547

Crompton R W 1969 Adv. Electr. Electron Phys. 271

Crompton R W, Elford M T and McIntosh A I 1968 Aust. J. Phys. 2143

Crompton R W and Morrison M A 1993 Aust. J. Phys. 46203

Crompton R W and Robertson A G 1971 Aust. J. Phys. 24543

Engelhardt A G and Phelps A V 1963 Phys. Rev. 1312115

England J P, Elford M T and Crompton R W 1988 Aust. J. Phys. 41573

Fujiwara T, Shimada T and Sugita K 1983 J. Phys. D: Appl. Phys. 161217

Ganguly B N and Garscadden A 1991 J. Appl. Phys. 70621

Gibson D K 1970 Aust. J. Phys. 23683

Gundersen M A and Schaefer F (eds) 1990 Physics and Applications of Pseudosparks NATO ASI Series B 219 (New York: Plenum)

Hall B I H 1955 Aust. J. Phys. 8468

Haydon S 1984 Personal communication

de Heer F J 1981 Phys. Scr. 23170

Henry R J W and Lane N F 1971 Phys. Rev. A 4410

Hiskes J R 1980 J. Appl. Phys. 514592

Huxley L G H and Crompton R W 1974 The Drift and Diffusion of Electrons in Gases (Wiley: New York)

Huxley L G H, Crompton R W and Elford M T 1966 Bull. Inst. Phys. Phys. Soc. July pp 251

Kokubo T, Tochikubo F and Makabe T 1989 J. Phys. D: Appl. Phys. 221281

Kuroda T and Iwakuro H 1993 Japan. J. Appl. Phys. 32 L1273

Kushner M J 1988 J. Appl. Phys. 632532

Lowke J J 1973 Aust. J. Phys. 26469

Lowke J J and Parker J H Jr 1969 Phys. Rev. 181302

Mclntosh A I 1966 Aust. J. Phys. 19805

Morgan W L 1992 Plasma Chem. Plasma Proc. 12 449, 477

Morgan W L 1993 Personal communication
Morrison M A, Crompton R W, Saha B C and Petrovic Z Lj 1987 Aust. J. Phys. 40239

Pack J L, Voshall R E and Phelps A V 1962 Phys. Rev. 127 2084

Petrović Z Lj 1985 PhD Thesis Australian National University (unpublished)

Petrović Z Lj and Crompton R W 1989 Aust. J. Phys. 42609

Petrovic Z Lj, Jelenkovic B M and Phelps A V 1992 Phys. Rev. Lett. 68325

Petrovic Z Lj and Phelps A V 1993 Phys. Rev. E 472806

Phelps A V, Petrović Z Lj and Jelenkovic B M 1993 Phys. Rev. E 472825

Rhymes T $1976 P h D$ Thesis Australian national University, unpublished

Robertson A G 1971 Aust. J. Phys. 24445

Robson R E 1972 Aust. J. Phys. 25685

Rose D J 1956 Phys. Rev. 104273

Roznerski W 1979 J. Phys. D: Appl. Phys. 11 L 197

Roznerski W and Leja K 1980 J. Phys. D: Appl. Phys. 13 L181 1984 J. Phys. D: Appl. Phys. 17279

Roznerski W, Leja K and Petrovic Z Lj 1989 Proc. 5th Int. Swarm Seminar, Birmingham, UK eds N Adams and D Smith p 45

Roznerski W and Mechlińska-Drewko J 1977 Rep ZNPG Fizyka XIX (Technical University of Gdansk) 264105

1979 J. Phys. D: Appl. Phys. $12 \mathrm{~L} 127$

Roznerski W, Mechlińska-Drewko J and Leja K $1990 \mathrm{~J}$. Phys. D: Appl. Phys. 231461

Schaefer G, Kristiansen M and Guenther A (eds) 1990 Gas Discharge Closing Switches (New York: Plenum)

Schmidt B 1993 Comm. At. Mol. Phys. 28379

Stokić Z, Fraga M M F R, Božin J, Stojanovic V, Petrović Z Lj and Jelenkovic B M 1992 Phys. Rev. A 457463

Tom̌ik B, Radovanov S, Petrovic Z Lj and Jelenkovic B M 1987 Proc. 8th Int. Symp. on Plasma Chemistry (Tokyo) 1987 p 1726

Vrhovac S B, Radovanov S B, Bzenic S A, Petrovic Z Lj and Jelenkovic B M 1991 Chem. Phys. 153233

Warten R W and Parker J H 1962 Phys. Rev. 1282661

Yoshida S, Phelps A V and Pitchford L C 1983 Phys. Rev. A 27 2858 